\title{
CYLINDRICITY OF ISOMETRIC IMMERSIONS BETWEEN HYPERBOLIC SPACES
}

BY

\author{
S. ALEXANDER AND E. PORTNOY
}

\begin{abstract}
The motivation for this paper was to prove the following analogue of the Euclidean cylinder theorem: any umbilic-free isometric immersion $\eta: H^{n-1} \rightarrow H^{n}$ between hyperbolic spaces takes the form of a hyperbolic $(n-2)$-cylinder over a uniquely determined parallelizing curve in $\bar{H}^{n}$. Our approach is through the more general study of isometric immersions generated by one-parameter families of hyperbolic $k$-planes without focal points. A by-product of this study is a natural extension to curves in $\bar{H}^{n}$ of the notion of a parallel family of $k$-planes along a curve in $H^{n}$; the extension is based on spherical symmetry of variation fields. Existence and uniqueness properties of this extended notion of parallelism are considered.
\end{abstract}

By a well-known theorem of Hartman and Nirenberg, every isometric immersion $\eta: E^{n-1} \rightarrow E^{n}$ between Euclidean spaces takes the form of a Euclidean $(n-2)$-cylinder [2]. That is, $\eta\left(E^{n-1}\right)$ may be obtained by parallel translation of an $(n-2)$-plane in $E^{n}$ along a base curve, where infinitely many choices of base curve are clearly possible. The analogous problem of determining the isometric immersions $\eta: H^{n-1} \rightarrow H^{n}$ between hyperbolic spaces of curvature -1 was posed by Nomizu in [4]. One may modify this problem by considering only the immersions which are umbilic-free, that is, have nowhere vanishing second fundamental form. Such an immersion is well known to be generated by hyperbolic $(n-2)$-planes (complete, $(n-2)$ dimensional, totally geodesic submanifolds) in $H^{n}$ [5]. The umbilic-free isometric immersions of $H^{n-1}$ into $H^{n}$ were characterized by Ferus in [1]. Ferus' analysis is concerned with the orthogonal trajectories of the generators (for example, see Theorem 3 below), and does not touch on the question of parallelization.

This paper studies isometric immersions $\eta: M \rightarrow H^{n}$ generated by oneparameter families, without focal points, of hyperbolic $k$-planes. For such an immersion, the sectional curvatures of $M$ satisfy $K_{M}<-1$; if $K_{M}=-1$ then $M$ is isometric to an open subset of $H^{k+1}$. The original motivation for this study was to prove Theorem 6 , which states that any umbilic-free isometric immersion of $H^{n-1}$ into $H^{n}$ takes the form of a hyperbolic $(n-2)$-cylinder

Received by the editors September 20, 1976.

AMS (MOS) subject classifications (1970). Primary 53C40; Secondary 53A35, $50 C 05$. 
over a uniquely determined parallelizing curve in $\bar{H}^{n}$ (see further remarks below). Here $\bar{H}^{n}=H^{n} \cup H_{\infty}^{n}$, where $H_{\infty}^{n}$ denotes the equivalence classes of asymptotic geodesic rays in $H^{n}$. This theorem offers an interesting analogue to the Euclidean cylinder theorem. The possibility of such an analogue was suggested by the classification of developable surfaces in $H^{3}$, Theorem 2, [6].

In $\S 1$, for any immersion $\eta: M \rightarrow H^{n}$ generated by a family of hyperbolic $k$-planes, the existence of a uniquely determined striction curve is obtained (Theorem 1). The striction point on a given $k$-plane is defined in terms of the variation field of the family; this is a vector field defined on and orthogonal to the $k$-plane, and "tangent to the family". If furthermore $K_{M}=-1$, it is shown that the striction curve is also the unique parallelizing curve in $\bar{H}^{n}$ of the family, so that the immersion takes the form of a hyperbolic $k$-cylinder (Theorem 2).

Here it is necessary to extend to curves in $\bar{H}^{n}$, the obvious notion of a parallel family of hyperbolic $k$-planes along a curve in $H^{n}$. This is done in a natural way, by proving first that a family $\left\{H_{c}\right\}_{c \in I}$ of $k$-planes is parallel along a regular curve $\sigma: I \rightarrow H^{n}$ if and only if for each $c$, the variation field is covariantly constant on the metric spheres in $H_{c}$ about $\sigma(c)$ (Proposition 1). Then the extension to curves $\sigma: I \rightarrow \bar{H}^{n}$ is obtained simply by substituting "horospheres" for "metric spheres" if $\sigma(c) \in H_{\infty}^{n}$.

$\$ 2$ considers foliations of $H^{n}$ by $(n-1)$-planes, and contains a criterion for generating such a foliation by parallel translation along a curve in $H^{n}$ (Theorem 4). $\$ 3$ studies isometric immersions of $H^{k+1}$ into $H^{n}$ generated by families of $k$-planes. For example, it is shown that any parallel family $\left\{H_{c}\right\}$ along a unit-speed curve $\sigma: R \rightarrow H^{n}$, for which the angle between the family and $\sigma$ is bounded away from zero, generates an immersion of $H^{k+1}$ into $H^{n}$ (Theorem 5). Umbilic-free isometric immersions of $H^{n-1}$ into $H^{n}$ are also discussed in $\$ 3$.

$\$ 4$ examines the implications of the extension to curves in $\bar{H}^{n}$ of the notion of parallel $k$-plane families. A differentiable manifold-with-boundary structure is placed on $\bar{H}^{n}$, namely that induced by the projective model of $H^{n}$. Along a regular curve $\sigma$ lying on $H_{\infty}^{n}$, the parallel translation problem has a unique solution for any allowable initial condition (Theorem 8). Of course, unique solutions always exist when $\sigma$ lies in $H^{n}$. Solutions do not generally exist if $\sigma$ takes both finite and infinite values (the question concerns piecing together solutions of two different problems, because of constraints which operate at $H_{\infty}^{n}$ ). However, if a solution exists, it is unique (Corollary 4).

The method of $\S 4$ is to convert the study of hyperbolic $k$-plane families into the study of Euclidean $k$-plane families, by using the projective model of $H^{n}$. Instead of the parallelizing curve of a hyperbolic family, one may examine the family of focal sets of a Euclidean family (Theorem 7). The classical 
approach to focal families (that is, to characteristics and envelopes) seems to be highly computational. Our approach is concise, coordinate-free, and new as far as we know.

Finally, in illustration of the preceding section, $\$ 5$ examines families of hyperbolic $k$-planes having parallelizing curves in $\bar{H}^{n}$ of certain special types.

Our conventions are as follows. On $H^{n}, g$ or $\langle$,$\rangle denotes the Riemannian$ metric, $\nabla$ denotes covariant differentiation, and $D$ denotes distance. Geodesics are always parametrized by arc-length. In $\$ \S 1-3$, no differentiable structure is assumed for $\bar{H}^{n}$. A curve is simply a map of an interval unless otherwise described. "Corollary 1.1" refers to part 1 of Corollary 1.

1. Families of $k$-planes in $H^{n}$. Let $\left(T_{1}, \ldots, T_{k}\right)$ be a smooth map of an open interval $I$ into the bundle of $k$-frames on $H^{n}$, and $H_{c}$ be the hyperbolic $k$-plane tangent to $\left(T_{1}, \ldots, T_{k}\right)(c)$ for each $c \in I$. Then the parametrized $k$-planes $\left\{H_{c}\right\}_{c \in I}$ will be called a (one-parameter) family in $H^{n}$. The map $\left(T_{1}, \ldots, T_{k}\right)$ will be called a frame for the family, and its projection $\alpha=$ $\pi \circ\left(T_{1}, \ldots, T_{k}\right): I \rightarrow H^{n}$, a base curve.

Such a family of hyperbolic $k$-planes determines a variation vector field $V_{c}$ on each of its members $H_{c}$, defined as follows: $V_{c p}$ is the component of $\beta^{\prime}(c)$ orthogonal to $H_{c}$, for any smooth curve $\beta$ in $H^{n}$ satisfying $\beta(c)=p$ and $\beta(c+\delta) \in H_{c+\delta},|\delta|<\varepsilon$. To see that the orthogonal components for all such curves $\beta$ agree, one may observe that if any one such $\beta$ is transverse to $H_{c}$ at $p$, then the $H_{c+\delta}$ locally determine a $(k+1)$-dimensional submanifold $M(p)$ through $p$ in $H^{n}$, and are the level hypersurfaces of a regular function $x$ on $M(p)$. Since by assumption every $\beta$ satisfies $x \cdot \beta=i d$, then with respect to the $H^{n}$-induced metric on $M(p)$, for every $\beta$ the component of $\beta^{\prime}(c)$ orthogonal to the level hypersurface $H_{c}$ is $\|\operatorname{grad} x\|^{-2} \operatorname{grad} x$.

The zeroes of the variation field $V_{c}$ form the focal set at $c$ of the family $\left\{H_{c}\right\}$. The family is regular at $c$ if $V_{c}$ does not vanish identically.

Given a frame for the family, define $\eta: I \times \mathbf{R}^{k} \rightarrow H^{n}$ by

$$
\eta\left(c, c_{1}, \ldots, c_{k}\right)=\exp \sum_{i=1}^{k} c_{i} T_{i}(c) .
$$

Denote by $x, x_{i}$ and $X, X_{i}$ respectively the coordinate functions and vector fields on $I \times \mathbf{R}^{k}$. Then $\eta_{*} X$ at $x=c$ is an $H^{n}$-Jacobi field on $H_{c}$ (that is, Jacobi along every geodesic in $H_{c}$ ). Since by definition, $V_{c}$ coincides with the component of $\eta_{*} X$ orthogonal to $H_{c}$, it follows from the form of Jacobi fields in $H^{n}$ that $V_{c}$ is Jacobi. Therefore the length function

$$
l_{c}=\left\|V_{c}\right\|
$$

on $H_{c}$ (carrying the $H^{n}$-induced metric) is convex, and strictly convex where nonvanishing. Since $H_{c}$ is isometric to $H^{k}$, the following lemma applies: 
LEMma 1. For any convex function $f: H^{k} \rightarrow \mathbf{R}$, the limit of $f$ along a geodesic ray in a fixed asymptote class is independent of the choice of ray.

Proof. By convexity, $f \circ \gamma$ has a limit in $[-\infty, \infty]$ along any geodesic ray $\gamma$. For $j=1,2$, suppose $\lim f \circ \gamma_{j}=n_{j}$, where $n_{1} \leqslant n_{2}$ and $\gamma_{1}$ and $\gamma_{2}$ are distinct asymptotic rays. Choose sequences $\left\{p_{1 i}\right\}$ and $\left\{p_{2 i}\right\}$ diverging to infinity along $\gamma_{1}$ and $\gamma_{2}$ respectively, and a point $q$ in the hyperbolic 2-plane containing $\gamma_{1}$ and $\gamma_{2}$, such that $p_{2 i}$ lies on the geodesic segment $\delta_{i}$ joining $p_{1 i}$ and $q$. Then $D\left(p_{1 i}, p_{2 i}\right)$ is bounded while $D\left(p_{2 i}, q\right)$ becomes arbitrarily large. Since $f \circ \delta_{i}$ is convex, $n_{1}<n_{2}$ would contradict finiteness of $f(q)$. Therefore $n_{1}=n_{2}$, as required.

Now let the variation function $\bar{l}_{c}: \bar{H}_{c} \rightarrow[0, \infty]$ be the extension, whose existence is guaranteed by Lemma 1 , of the length function given by (2).

Lemma 2. If a family $\left\{H_{c}\right\}$ of $k$-planes in $H^{n}$ has no focal points at $c$, then the variation function at $c$ has a single minimum point, which is the limit as $\delta \rightarrow 0$ of the point of $\bar{H}_{c}$ closest to $H_{c+\delta}$.

Proof. It is a consequence of strict convexity that $l_{c}$ has at most one critical point and $\bar{l}_{c}$ has exactly one minimum point. (If $\bar{l}_{c}$ is finite at a point $p \in H_{c \infty}$, then $l_{c}$ must decrease on all geodesic rays to $p$.)

For $p \in H_{c}$, let $\beta_{p}(c+\delta)$ be the point of $H_{c+\delta}$ nearest $p$. Then it is not hard to see that $\beta_{p}=\exp _{p} k N$ for smooth function $k$ and vector-valued function $N$, where $k(c)=0$ and $N(c) \perp H_{c}$. Since $\beta_{p}^{\prime}(c)=k^{\prime}(c) N(c)$, which is orthogonal to $H_{c}$, then $V_{c p}=\beta_{p}^{\prime}(c)$. It follows that

$$
l_{c}(p)=\lim _{\delta \rightarrow 0^{+}}\left[D\left(p, H_{c+\delta}\right) / \delta\right] .
$$

The function $D\left(\cdot, H_{c+\delta}\right)$ on $H_{c}$ is strictly convex, and therefore extends to $\bar{H}_{c}$ with exactly one minimum point $p_{\delta}$. From (3) and the fact that convergence of convex functions on Riemannian manifolds is uniform on compact subsets, we may conclude easily that $p_{\delta}$ converges to the minimum point of $\bar{l}_{c}$, as was claimed. This completes the proof of Lemma 2.

Next suppose $\left\{H_{c}\right\}_{c \in I}$ is a family without focal points. Let $M=\{(c, p)$ : $\left.c \in I, p \in H_{c}\right\}$, and define $\eta: M \rightarrow H^{n}$ by $\eta(c, p)=p$. A frame $\left(T_{1}, \ldots, T_{k}\right)$ for the family determines an obvious coordinatization of $M$ by $I \times R^{k}$, for which $\eta$ is expressed by (1); and thus determines a differentiable structure on $M$, with respect to which $\eta$ is an immersion. This structure is independent of choice of frame. Letting $M$ carry the $\eta$-induced Riemannian metric, we say that the isometric immersion $\eta: M \rightarrow H^{n}$ is generated by the family, and has generators $H_{c}$. In what follows, we shall sometimes suppress $\eta$, identifying $M$ locally with its image in $H^{n}$.

Observe that by the Gauss equation, sectional curvatures of $M$ satisfy $K_{M} \leqslant-1$, with equality holding if and only if the normalized variation field 
$l_{c}^{-1} V_{c}$ is covariantly constant on $H_{c}$ for all $c \in I$.

For each $c \in I$ the variation function $i_{c}$ has a unique minimum point, which by Lemma 2 it is reasonable to call the striction point. Note that the variation function on a given $k$-plane depends only up to a constant multiple on the choice of regular parametrization of the family (equivalently, of the base curve $\alpha$ ), so the striction point is independent of that choice. If $\bar{l}_{c}(p)=0$ for some $p \in H_{c \infty}$, we say the family is asymptotic at $p$; this terminology is justified by (3).

THEOREM 1. Let $\eta: M \rightarrow H^{n}$ be the isometric immersion generated by a family $\left\{H_{c}\right\}_{c \in I}$ of hyperbolic $k$-planes without focal points. Then there is a uniquely determined curve $\sigma: I \rightarrow \bar{H}^{n}$ which satisfies: (i) $\sigma(c) \in \bar{H}_{c}$ for all $c \in I$; (ii) the generators are $M$-parallel along $\sigma \mid J$ when $\sigma \mid J$ lies in $H^{n}$; (iii) the generators are asymptotic at $\sigma(c)$ when $\sigma(c) \in H_{\infty}^{n}$. The curve $\sigma$ coincides with the curve of striction points.

Proof. Note that here we regard $\bar{H}_{c}$ as lying in $\bar{H}^{n}$, in the natural way. Given $c_{0} \in I$, it suffices to prove that the theorem holds for some open subinterval about $c_{0}$. Therefore there is no loss of generality in assuming that $M$ is coordinatized with respect to a base curve $\alpha$ orthogonal to the $H_{c}$. Thus we assume $M$ carries coordinate vector fields $X, X_{1}, \ldots, X_{k}$ for which (supressing $\eta$ and $\eta_{*}$ ) the $X_{i}$ are tangent to the $H_{c}$; the restriction of $X$ to each $H_{c}$ is Jacobi in $H^{n}$; along $\alpha, X=\alpha^{\prime}$ and the $X_{i}$ are covariantly constant in the normal bundle of $\alpha$ in $M$. Let $\gamma$ be a geodesic in $H_{c}$ satisfying $\gamma(0)=\alpha(c)$. By parallelism of $X_{i}, \nabla_{\alpha^{\prime}(c)}\left(\Sigma c_{i} X_{i}\right)$ is orthogonal to $H_{c}$, where $\gamma^{\prime}(0)=\Sigma c_{i} X_{i \alpha(c)}$ and $\nabla$ is covariant differentiation in $H^{n}$. Therefore $\nabla_{\gamma^{\prime}(0)} X$ is orthogonal to $H_{c}$. Since $X$ is $H^{n}$-Jacobi along $\gamma, X$ is orthogonal to $H_{c}$ along $\gamma$. Therefore by the definition of variation field,

$$
X \mid H_{c}=V_{c}
$$

and at any point of $H_{c}$,

$$
\left\langle\nabla_{X} X_{i}, X\right\rangle=\left\langle\nabla_{X_{i}} X, X\right\rangle=\left(X_{i} l_{c}\right) l_{c} .
$$

Now let $\sigma(c)$ denote the striction point at $c$. If $\sigma \mid J$ lies in $H^{n}$ then $\sigma \mid J$ is smooth (this follows from the implicit function theorem and the fact that $\sigma(c)$ is a nondegenerate critical point of $l_{c}$ ). Since $\left\{H_{c}\right\}$ has no focal points, $\sigma \mid J$ is regular. By (5), since $\sigma(c)$ is a critical point of $l_{c}$ and $H_{c}$ is totally geodesic,

$$
\left\langle\nabla_{\sigma^{\prime}} X_{i}, X\right\rangle=\left\langle\nabla_{X} X_{i}, X\right\rangle=0 \text {. }
$$

Therefore the tangent $k$-planes to the $H_{c}$ are $M$-parallel along $\sigma \mid J$, as required. Conversely, if $\sigma^{*}: J^{*} \rightarrow H^{n}$ is any curve in $H^{n}$ satisfying $\sigma^{*}(c+\delta)$ $\in H_{c+\delta}$ and parallelizing the $H_{c}$ in $M$, then $\sigma^{*}(c)$ is critical for $l_{c}$, so $\sigma^{*}(c)=\sigma(c)$ for all $c \in J^{*}$. 
By the Jacobi equation, if $U_{1}, \ldots, U_{n-k}$ are covariantly constant orthonormal fields defined on and orthogonal to $H_{c}$, then

$$
V_{c}=\sum_{j=1}^{n-k} h_{j} U_{j}
$$

where the $h_{j}$ are hyperbolic functions on $H_{c}$, that is, where $\left(h_{j} \circ \gamma\right)^{\prime \prime}=h_{j} \circ \gamma$ for every geodesic $\gamma$ in $H_{c}$. If $\gamma(0)=q, V_{c q}=\Sigma a_{j} U_{j q}$ and $\nabla_{\gamma^{\prime}(0)} V_{c}=\Sigma b_{j} U_{j q}$, then

$$
h_{j} \circ \gamma(s)=a_{j} \cosh s+b_{j} \sinh s, \quad 1<j<n-k .
$$

If the striction point $\sigma(c)$ lies at $H_{\infty}^{n}$ and $\gamma$ is a geodesic ray to $\sigma(c)$, the limit of $h_{j} \circ \gamma$ is finite for all $j$, by definition of $\sigma(c)$. But then $a_{j}=-b_{j}$ in (7), and the limit of $h_{j}$ along $\gamma$ vanishes for all $j$. Therefore $\bar{l}_{c}(\sigma(c))=0$. Conversely, if $i_{c}(p)=0$ for some $p \in H_{c \infty}$, obviously $p$ is the striction point at $c$. This completes the proof of Theorem 1.

The proof of Theorem 1 has the following corollary, of which the second part is essentially due to Ferus [1]:

COROLlaRY 1. Let $\eta: M \rightarrow H^{n}$ be the isometric immersion generated by a family $\left\{H_{c}\right\}$ of hyperbolic $k$-planes without focal points. 1 . The curvature vector in $M$ of an orthogonal trajectory to the generators at any $q \in H_{c}$ is $\left(-l_{c}^{-1} \operatorname{grad} l_{c}\right)_{q}$.

2. If $M$ has constant sectional curvature -1 , then the curvature in $M$ of such a curve does not exceed 1 .

Proof. 1. Our notation will be that of the preceding proof. Since $X$ is orthogonal to the $H_{c}$, the curvature vector in question is the orthogonal projection tangent to $H_{c}$ of $\|X\|^{-2} \nabla_{X} X$ at $q$. But by (5) and (4),

$$
\left\langle\|X\|^{-2} \nabla_{X} X, X_{i}\right\rangle=-l_{c}^{-1} X_{i} l_{c}=\left\langle-l_{c}^{-1} \operatorname{grad} l_{c}, X_{i}\right\rangle .
$$

2. If $M$ has constant sectional curvature -1 , then in (6) we may take $h_{1}=l_{c}$ and $h_{j}=0$ for $j>1$. Thus $l_{c} \circ \gamma(s)=a \cosh s+b \sinh s$ for any geodesic $\gamma$ in $H_{c}$, by (7), where $\left|a^{-1} b\right| \leqslant 1$ since $l_{c}>0$. If $\gamma$ is chosen so that

$$
\gamma(0)=q \text { and } \gamma^{\prime}(0)=\left(-\left\|\operatorname{grad} l_{c}\right\|^{-1} \operatorname{grad} l_{c}\right)_{q},
$$

then $a=l_{c}(q), b=-\left\|\operatorname{grad} l_{c}\right\|(q)$, and $\left|a^{-1} b\right|$ is the required curvature at $q$ by part 1 .

REMARK 1. By Corollary 1.1, an orthogonal trajectory is an $M$-geodesic if and only if it coincides with the striction curve.

Proposition 1. A family $\left\{H_{c}\right\}$ of hyperbolic $k$-planes is $H^{n}$-parallel along an arbitrary regular base curve $\sigma$ in $H^{n}$ if and only if for each $c$, the variation field at $c$ is covariantly constant on metric spheres in $H_{c}$ about $\sigma(c)$. 
Proof. If $V_{c}$ is covariantly constant on metric spheres about some $q \in H_{c}$, then for $h_{j}$ as in (6), $\left(h_{j} \circ \gamma\right)^{\prime}(0)=0$ for any geodesic $\gamma$ in $H_{c}$ with $\gamma(0)=q$; hence $b_{j}=0$ for all $j$, in (7). It follows that $V_{c}$ is covariantly constant on metric spheres in $H_{c}$ about $q$ if and only if

$$
V_{c p}=a \cosh D(p, q) U_{p}
$$

where $U$ is a covariantly constant unit normal to $H_{c}$.

Now suppose $V_{c o(c)} \neq 0$. Locally the family generates an imbedded submanifold which, as in the proof of Theorem 1, carries coordinate vector fields $X, X_{1}, \ldots, X_{k}$. Here the restriction of $X$ to each member of the family coincides with the variation field, the $X_{i}$ are tangent to the family, and (5) is satisfied. If $V_{c}$ is covariantly constant on spheres about $\sigma(c)$, then $\sigma(c)$ is critical for the length function $l_{c}$. Furthermore, on $H_{c}, \nabla_{X_{i}} X$ is proportional to $X$ by (8). Therefore $\nabla_{X} X_{i}=0$ at $\sigma(c)$ by (5), so that $\nabla_{\sigma^{\prime}(c)} X_{i}$ is tangent to $H_{c}$, as required. Conversely, if $\nabla_{\sigma^{\prime}(c)} X_{i}$ is tangent to $H_{c}$, then so is $\nabla_{X} X_{i}$ at $\sigma(c)$, since $\sigma^{\prime}(c)$ is transverse to $H_{c}$ by assumption. Since $\left\langle\nabla_{X_{j}} X, X_{j}\right\rangle=$ $-\left\langle\nabla_{X_{i}} X_{j}, X\right\rangle=0$, it follows that $\nabla_{X_{i}} X=0$ at $\sigma(c)$. Therefore by (7), $V_{c}$ is covariantly constant on spheres about $\sigma(c)$.

Suppose instead that $V_{c o(c)}=0$. Define a map $\eta: I \times \mathbf{R}^{k} \rightarrow H^{n}$ by (1), for any choice of frame over $\sigma$. It suffices to show that $V_{c}$ is covariantly constant on spheres about $\sigma(c)$ if and only if if the $k$ vectors

$$
Y_{i}=\nabla_{\sigma^{\prime}(c)}\left(\left.\eta_{*} X_{i}\right|_{x^{\prime}-\cdots=x^{k}=0}\right)=\nabla_{\eta_{*} X_{i}}\left(\left.\eta_{*} X\right|_{x=c}\right) \text { at } \sigma(c)
$$

are tangent to $H_{c}$. If $V_{c}$ is constant on spheres about $\sigma(c)$, then $V_{c}$ vanishes identically, by (8). Since $V_{c}$ is the component of $\left.\eta_{*} X\right|_{x=c}$ orthogonal to $H_{c}$, it follows that the $Y_{i}$ are tangent to $H_{c}$. Conversely, if the $Y_{i}$ are tangent to $H_{c}$, then for any geodesic $\gamma$ with $\gamma(0)=\sigma(c)$, we have $b_{j}=0$ in (7). Since $a_{j}=0$ by assumption, $V_{c}$ vanishes identically. Thus Proposition 1 is proved.

We shall call a family $\left\{H_{c}\right\}_{c \in I}$ of hyperbolic $k$-planes parallel in $\bar{H}^{n}$ along a curve $\sigma: I \rightarrow \bar{H}^{n}$ if for each $c, \sigma(c) \in \bar{H}_{c}$, and the variation field at $c$ is covariantly constant on metric spheres about $\sigma(c)$ if $\sigma(c) \in H_{c}$, or on horospheres about $\sigma(c)$ if $\sigma(c) \in H_{c \infty}$. (In the latter case, the variation function will be shown to vanish at $\sigma(c)$.) By Proposition 1, this definition is simply an extension of the usual one.

Proposition 2. Let $\eta: M \rightarrow H^{n}$ be the isometric immersion generated by a family $\left\{H_{c}\right\}_{c \in I}$ without focal points, and let $\sigma: I \rightarrow \bar{H}^{n}$ be the striction curve of the family. Then $M$ has constant sectional curvature -1 if and only if the family is parallel in $\bar{H}^{n}$ along $\sigma$.

Proof. Recall that $K_{M}=-1$ if and only if for each $c, l_{c}^{-1} V_{c}$ is covariantly constant. We claim that this occurs if and only if $V_{c}$ is covariantly constant on spheres about $\sigma(c)$. Indeed, if $\sigma(c) \in H_{c}$ and $V_{c}$ is constant on metric 
spheres about $\sigma(c)$, then $l_{c}^{-1} V_{c}$ is constant by (8). If $\sigma(c) \in H_{\infty}^{n}$, then $l_{c}^{-1} V_{c}$ is constant on each geodesic ray to $\sigma(c)$ by the remarks following (7); thus constancy on horospheres about $\sigma(c)$ implies constancy on all of $H_{c}$.

For the converse, consider any $H^{n}$-Jacobi field on $H_{c}$ of the form $l U$, where $l>0, U$ is a given covariantly constant unit normal to $H_{c}$, and $l$ has a given value at some fixed point of $H_{c}$. Such a field is determined by choice of minimum point $q \in \bar{H}_{c}$ for $l$, since $l$ is hyperbolic. Since $l$ is a constant multiple of $\cosh D(\cdot, q)$ when $q$ is finite, then $l$ is constant on metric spheres about $q$ when $q \in H_{c}$, and by a limit argument, on horospheres about $q$ when $q \in H_{c \infty \infty}$.

THEOREM 2. A regular family $\left\{H_{c}\right\}_{c \in I}$ of hyperbolic $k$-planes is without focal points and generates an immersed submanifold of constant curvature -1 if and only if the family is parallel in $\bar{H}^{n}$ along some curve $\sigma: I \rightarrow \bar{H}^{n}$. If such a parallelizing curve $\sigma$ exists, it is unique.

Proof. It suffices, by Proposition 2, to show that if the family is regular and parallel in $\bar{H}^{n}$ along some $\sigma$, then there are no focal points and $\sigma$ is the striction curve of the family. But if $V_{c}$ is covariantly constant on metric spheres about some $q \in H_{c}$, then $V_{c}$ is given by (8). Thus $V_{c}$ never vanishes because it does not vanish identically, and $q$ is the striction point at $c$. If $V_{c}$ is constant on horospheres about $q \in H_{c \infty}$, then again $V_{c}$ never vanishes, because otherwise the zero set of $V_{c}$ would be a hyperbolic $r$-plane for $0<r<k$, and no such $r$-plane contains a horosphere of $H_{c}$. Furthermore, since the striction point is the unique minimum point of the length function $\bar{l}_{c}$, this point is certainly $q$.

REMARK 2. A submanifold of the type considered in Theorem 2 is always isometric to an open subset of $H^{k+1}$ (see Proposition 3).

RemarK 3. We shall see in $\S 4$ that if $\bar{H}^{n}$ is given the manifold-withboundary structure induced by the projective model of $H^{n}$, then the parallelizing curve $\sigma$ of Theorem 2 is smooth in $\bar{H}^{n}$. Furthermore, when $\sigma$ lies at infinity there are constraints on the derivatives of $\sigma$. For example, if $k=1, \sigma^{\prime}$ vanishes at infinity (Corollary 2); thus if $\sigma$ lies at infinity on an interval, it is constant there, and the immersion takes the form of an asymptotic cone.

2. Foliations of $H^{n}$ by hyperplanes. Let $\mathscr{F}$ denote a foliation of $H^{n}$ by hyperbolic $(n-1)$-planes. We shall require the following important theorem of Ferus concerning such foliations:

THEOREM 3 [1]. 1. For any $p \in H^{n}$ and any regular curve $\alpha$ in $H^{n}$ with curvature $\kappa<1$, the function $D(\alpha(t), p)$ has at most one critical point, namely a strict relative minimum point, and is unbounded if $\alpha$ has infinite length. In consequence: 
2. The family of orthogonal hyperplanes to a regular curve with $\kappa<1$ is without self-intersections.

3. For a regular curve with $\kappa \leqslant 1$ and unbounded length in both directions, the family of orthogonal hyperplanes foliates $H^{n}$.

REMARK 4. A family $\left\{H_{c}\right\}$ of hyperplanes in $H^{n}$ is without focal points if and only if it is without self-intersections. Corollary 1.2 and Theorem 3.2 imply that if there are no focal points then there are no self-intersections. On the other hand, since the variation field for an arbitrary hyperplane family, possibly with focal points, is of the form $h U$ where $U$ is covariantly constant and $h$ is hyperbolic, the focal set in $H_{c}$ is nonempty only if it is an $(n-2)$-plane on either side of which $h$ takes opposite signs. Observing that $h$ is given by the expression in (3), where $D$ is interpreted as directed distance toward $U$, one may show that each focal point is the limit of intersection points of $H_{c}$ and $H_{c+\delta}$ as $\delta \rightarrow 0$. Thus our remark is verified.

Given a foliation $\mathscr{F}$ of $H^{n}$ by hyperplanes, one always may choose a regular curve $\alpha: I \rightarrow H^{n}$ of infinite length which is everywhere orthogonal to $\mathscr{F}$. By Corollary 1.2 and Theorem 3.3, the family of hyperplanes orthogonal to $\alpha$ is all of $\mathcal{F}$. Therefore $\mathcal{F}$ corresponds to a regular one-parameter family $\left\{H_{c}\right\}_{c \in I}$ (without focal points) of hyperplanes in $H^{n}$, and by Theorem 2, possesses a uniquely determined parallelizing curve $\sigma: I \rightarrow \bar{H}^{n}$.

The following theorem gives a necessary and sufficient condition that a given curve $\sigma$ in $H^{n}$ and initial transverse hyperplane determine by parallel translation a foliation of $H^{n}$.

THEOREM 4. Let $\sigma: \mathbf{R} \rightarrow H^{n}$ be a unit-speed curve, $H_{0}$ a hyperbolic $(n-1)$ plane through $\sigma(0)$, and $H_{c}$ the parallel translate of $H_{0}$ along $\sigma$ to $\sigma(c)$.

1. $\left\{H_{c}\right\}$ is without focal points if and only if $H_{c}$ is transverse to $\sigma^{\prime}(c)$ for all $c \in \mathbf{R}$.

2. Assuming that $\left\{H_{c}\right\}$ is without focal points, let $\theta(c) \in(0, \pi / 2]$ be the angle between $H_{c}$ and $\sigma^{\prime}(c)$. Fix $q \in H^{n}$. Then $\left\{H_{c}\right\}$ foliates $H^{n}$ if and only if

$$
\int_{0}^{\infty} f(x) d x=\infty \text { and } \int_{-\infty}^{0} f(x) d x=\infty \text {, }
$$

where $f(x)=\sin \theta(x) \cosh D(q, \sigma(x))$. In particular:

3. $\left\{H_{c}\right\}$ foliates $H^{n}$ if $\theta$ is bounded away from zero.

Proof. 1. By (8) and the definition of variation field, $V_{c p}=a \cdot$ $\cosh D(p, \sigma(c)) U_{p}$, where $U$ is a covariantly constant unit normal to $H_{c}$ and $a=\left\langle\sigma^{\prime}(c), U\right\rangle=\sin \theta(c)$. Thus $V_{c}$ is nowhere vanishing if $\theta(c) \neq 0$ and vanishes identically if $\theta(c)=0$.

2. Let $\alpha: I \rightarrow H^{n}$ be a maximal curve satisfying $\alpha(c) \in H_{c}$ and $\alpha^{\prime}(c)=$ $V_{c \alpha(c)}$ for all $c \in I$. Since $\left\{H_{c}\right\}_{c \in I}$ is without focal points and $\alpha$ is an 
orthogonal trajectory, the curvature of $\alpha$ does not exceed 1. The length of $\alpha$ is unbounded in both directions if and, by Theorem 3.3, only if $I=\mathbf{R}$ and $\left\{H_{c}\right\}$ foliates $H^{n}$.

Suppose $\left\{H_{c}\right\}$ does not foliate $H^{n}$. We may assume $\alpha$ has finite length in the positive direction. Finiteness and maximality of $\alpha$ imply that the domain of $\alpha$ includes $[d, \infty)$ for some $d$. Then

$$
\begin{aligned}
\left\|\alpha^{\prime}(x)\right\| & =\sin \theta(x) \cosh D(\alpha(x), \sigma(x)) \\
& \geqslant \sin \theta(x) \cosh [D(q, \sigma(x))-D(q, \alpha(x))] \\
& \geqslant L f(x)
\end{aligned}
$$

for some $L>0$ independent of $x \in[d, \infty)$, since $D(q, \alpha(x))$ is bounded on $[d, \infty)$. Therefore $\int_{d}^{\infty} f(x) d x<\infty$.

Now, suppose $\left\{H_{c}\right\}$ foliates $H^{n}$. Choose $\alpha$ to pass through $q$. By Theorem 3.1, $D(\alpha, \sigma(x))=D(\alpha(x), \sigma(x)) \leqslant D(q, \sigma(x))$ for each $x$. Therefore $\left\|\alpha^{\prime}(x)\right\|$ $\leqslant f(x)$, and since $\alpha$ has unbounded length in both directions, the required divergence of integrals follows.

EXAMPLE 1. Let $\sigma: \mathbf{R} \rightarrow H^{n}$ be any unit-speed curve with total curvature

$$
\int\left\|\nabla_{\mathbf{\sigma}^{\prime}} \boldsymbol{\sigma}^{\prime}\right\|<\pi
$$

with each of

$$
\int_{0}^{\infty}\left\|\nabla_{\sigma^{\prime}} \sigma^{\prime}\right\| \text { and } \int_{-\infty}^{0}\left\|\nabla_{\sigma^{\prime}} \sigma^{\prime}\right\|
$$

less than $\pi / 2$; and let $H_{0}$ be orthogonal to $\sigma$. Then the family of parallel translates foliates $H^{n}$.

Indeed, suppose $I$ is a maximal interval about 0 on which the family is transverse to $\sigma$, and let $U$ be the unit normal to the family along $\sigma \mid I$ in the direction of $\sigma^{\prime}(c)$. Then

$$
\left|\left\langle\sigma^{\prime}, U\right\rangle^{\prime}\right|=\left|\left\langle\nabla_{\sigma^{\prime}} \sigma^{\prime}, U\right\rangle\right| \leqslant\left\|\nabla_{\sigma^{\prime}} \sigma^{\prime}\right\| \cos \theta,
$$

whence $\left|\theta^{\prime}\right| \leqslant\left\|\nabla_{\sigma^{\prime}} \sigma^{\prime}\right\|$, where $\theta$ is the angle between $\sigma^{\prime}$ and $H_{c}$. Therefore our hypothesis implies that $\theta$ is bounded away from 0 on $I$. But then $I=\mathbf{R}$ and the claim follows by Theorem 4.3.

3. Isometric immersions of $H^{k+1}$ into $H^{n}$. First we take note of the global structure of an immersed submanifold of $H^{n}$ of the type considered in Theorem 2:

Proposition 3. Let $\eta: M \rightarrow H^{n}$ be the isometric immersion generated by a family of hyperbolic $k$-planes without focal points. If $K_{M}=-1$ then $M$ is isometric to an open submanifold of $H^{k+1}$.

Proof. Since $M$ and $H^{k+1}$ are analytic and locally isometric, and since $M$ 
is simply connected, there exists an isometric immersion $\iota: M \rightarrow H^{k+1}([3, \mathrm{pp}$. 255 and 263]). By assumption, $M$ is foliated by a family $\left\{\tilde{H}_{c}\right\}$ of complete, totally geodesic submanifolds (where $\left.\eta\left(\tilde{H}_{c}\right)=H_{c}\right)$. But then $\iota\left(\tilde{H}_{c}\right)$ is a $k$-plane in $H^{k+1}$ for each $c$. Since $\iota$ is an immersion, the family $\left\{\iota\left(\tilde{H}_{c}\right)\right\}$ is without focal points. By Remark 3, $\left\{\iota\left(H_{c}\right)\right\}$ is without self-intersections, and it follows that $\iota$ is one-one.

Remark 5. Consider an isometric immersion $\eta: M \rightarrow H^{n}$ such as is described in Proposition 3, and identify $M$ with an open submanifold of $H^{k+1}$. Then $M$ is foliated by a family $\left\{\tilde{H}_{c}\right\}$ of hyperbolic hyperplanes, where $\eta\left(\tilde{H}_{c}\right)=H_{c}$. Denote by $\tilde{\sigma}: I \rightarrow \bar{H}^{k+1}$ and $\sigma: I \rightarrow \bar{H}^{n}$ the parallelizing curves of the families $\left\{\tilde{H}_{c}\right\}$ and $\left\{H_{c}\right\}$ respectively (the existence of $\tilde{\sigma}$ and $\sigma$ is guaranteed by Theorem 2), and by $\eta_{c}: \overline{\left(\tilde{H}_{c}\right) \rightarrow \bar{H}_{c}}$ the natural identification extending $\eta \mid \tilde{H}_{c}$. If $\tilde{V}_{c}$ and $V_{c}$ are the variation fields at $c$ for the respective families, then $\eta_{*} \tilde{V}_{c}$ is orthogonal to $H_{c}$ since $\eta$ is isometric. Therefore $\eta_{*} \tilde{V}_{c}=V_{c}$, and $\eta_{c} \circ \tilde{\sigma}(c)=\sigma(c)$ for all $c \in I$.

The following construction of a class of isometric immersions of $H^{k+1}$ into $H^{n}$ depends upon Theorem 4:

THEOREM 5. Let $\sigma: \mathbf{R} \rightarrow H^{n}$ be a unit-speed curve, $H_{0}$ a hyperbolic $k$-plane through $\sigma(0)$, and $H_{c}$ the parallel translate of $H_{0}$ along $\sigma$ to $\sigma(c)$. Let $\theta(c)$ be the angle between $\sigma^{\prime}(c)$ and $H_{c}$, for each $c \in \mathbf{R}$. If $\theta$ is bounded away from zero, then the family $\left\{H_{c}\right\}$ generates an isometric immersion $\eta: H^{k+1} \rightarrow H^{n}$. The immersion $\eta$ is umbilic-free if and only if the curvature vector in $H^{n}$ of $\sigma$ at $c$ is transverse to the span of $\sigma^{\prime}(c)$ and $H_{c}$ for each $c \in \mathbf{R}$.

Proof. The family $\left\{H_{c}\right\}$ is regular since it is transverse to $\sigma$. Therefore by Theorem 2, $\left\{H_{c}\right\}$ generates an isometric immersion $\eta: M \rightarrow H^{n}$ where $M$ has curvature -1 . By Proposition 3, $M$ may be identified with an open submanifold of $H^{k+1}$. Since $\eta$ is an isometric immersion, we have a unit-speed curve $\tilde{\sigma}: \mathbf{R} \rightarrow M$, and a family $\left\{\tilde{H}_{c}\right\}$ of hyperbolic $k$-planes which foliates $M$ and is parallel along $\tilde{\sigma}$, and whose angle with $\tilde{\sigma}$ is bounded away from zero (where $\eta \circ \tilde{\sigma}=\sigma$ and $\eta\left(\tilde{H}_{c}\right)=H_{c}$ ). But then by Theorem 4.3, $\left\{\tilde{H}_{c}\right\}$ foliates $H^{k+1}$, and so $M=H^{k+1}$.

Let $K$ be the open submanifold of $H^{k+1}$ on which the nullity of the second fundamental form of $\eta$ takes its minimum value $\nu$. Since all tangent vectors to the $\tilde{H}_{c}$ are nullity vectors, $k<\nu<k+1$. Recall that the nullity foliation of $K$ is well known to have complete leaves: for the present situation, namely immersion of $H^{k+1}$ into $H^{n}$, completeness was proved in [5]. The transversality hypothesis on the curvature vectors of $\sigma$ implies that nullity on $K$ is $k$ and $\tilde{\sigma}$ lies in $K$. Therefore $K$ contains the complete $k$-plane $\tilde{H}_{c}$ for every $c \in \mathbf{R}$, and so $K=H^{k+1}$ and $\eta$ has no umbilics. Conversely, if there are no 
umbilics, then $\nu=k$ along $\tilde{\sigma}$, and the transversality condition follows. This completes the proof of Theorem 5 .

Finally we consider umbilic-free isometric immersions $\eta: H^{n-1} \rightarrow H^{n}$. For such an immersion, $H^{n-1}$ is foliated by hyperbolic $(n-2)$-planes tangent to the nullity spaces of the second fundamental form. As in the previous section, the foliating $(n-2)$-planes may be regarded as a regular one-parameter family $\left\{\tilde{H}_{c}\right\}_{c \in I}$ in $H^{n-1}$. Since $\eta$ is totally geodesic on each $\tilde{H}_{c}, \eta$ is generated by a family $\left\{H_{c}\right\}_{c \in I}$, without focal points, of $(n-2)$-planes in $H^{n}$. Then Theorem 2 immediately yields:

THEOREM 6. Let $\eta: H^{n-1} \rightarrow H^{n}$ be an umbilic-free isometric immersion and $\left\{H_{c}\right\}_{c \in I}$ be the family of generators of $\eta$. Then there is a uniquely determined curve $\sigma: I \rightarrow \bar{H}^{n}$ along which the generators are parallel in $\bar{H}^{n}$.

4. Parallelization at $H_{\infty}^{n}$. In order to study the behavior at infinity of the parallelizing curve of a family, we shall give $\bar{H}^{n}$ the differentiable manifoldwith-boundary structure of $\bar{D}^{n}$ induced by the projective model. By working with the projective model, we shift our attention to families of Euclidean $k$-planes in $E^{n}$ and their intersection properties. Although the methods of this section are Euclidean, note that all the results except Theorem 7 are results about $\bar{H}^{n}$ carrying the given differentiable structure.

$g^{*}$ or - will denote the standard Riemannian metric on $E^{n}$, and $D^{*}$ will denote Euclidean distance. Any family $\left\{H_{c}{ }^{*}\right\}$ of Euclidean $k$-planes in $E^{n}$ has a variation field $V_{c}^{*}$ at $c$, which is defined just as before but in terms of $g^{*}$, and which is a $g^{*}$-Jacobi field on $H_{c}^{*}$. Thus

$$
V_{c}^{*}=\sum_{j=1}^{n-k} h_{j}^{*} U_{j}^{*}
$$

where the $U_{j}^{*}$ are constant orthonormal fields defined on and orthogonal to $H_{c}{ }^{*}$, and the $h_{j}{ }^{*}$ are affine functions on $H_{c}{ }^{*}$. The focal set $F_{c}$ of $\left\{H_{c}^{*}\right\}$ at $c$ is the intersection of the zero sets of the $h_{j}^{*}$, and if nonempty is an $r$-plane (possibly "at infinity") for some $r \geqslant 2 k-n$. (Since the solution set of any homogeneous linear equation on a projective space is a projective hyperplane, we are justified in adopting the convention that any affine function on a Euclidean space vanishes on a hyperplane, which lies "at infinity" if the function is constant.) Since two affine functions are constant multiples of each other if and only if they have the same zeros, $F_{c}$ is a $(k-1)$-plane if and only if we may assume $h_{j}^{*}=0$ for all $j>1$ in (9).

For the rest of this section, $H^{n}$ will be identified with the Euclidean open unit disc $D^{n}$ carrying the projective hyperbolic metric $g$. Unparametrized $H^{n}$-geodesics are thus identified with line segments in $D^{n}$, and totally geodesic submanifolds of $H^{n}$ with totally geodesic submanifolds of $D^{n}$. 
Asymptotic geodesics in $H^{n}$ correspond to segments approaching the same point of $\partial D^{n}=S^{n-1}$. Thus $H_{\infty}^{n}$ is identified with $S^{n-1}$, and $\bar{H}^{n}$ with $\bar{D}^{n}$.

To each $k$-plane of a given family $\left\{H_{c}\right\}$ in $H^{n}$ may be associated the complete Euclidean $k$-plane $H_{c}^{*}$ satisfying $H_{c}=H_{c}^{*} \cap D^{n}$. The hyperbolic family $\left\{H_{c}\right\}$ is without focal points if and only if for each $c$, the focal set of $\left\{H_{c}{ }^{*}\right\}$ does not enter $D^{n}$. (Note that focal points depend only on differentiable structure, not on metric structure.) Such a family $\left\{H_{c}\right\}$ generates an immersed submanifold of $H^{n}$. The $g$-induced metric on this submanifold has curvature -1 if and only if for each $c$, the hyperbolic variation field $V_{c}$ is tangent to a fixed hyperbolic $(k+1)$-plane through $H_{c}$; hence if and only if the Euclidean variation field of $\left\{H_{c}^{*}\right\}$ is tangent to a fixed Euclidean $(k+1)$-plane through $H_{c}^{*}$. Equivalent conditions on $\left\{H_{c}^{*}\right\}$ are that the metric induced by $g^{*}$ on the submanifold generated by $\left\{H_{c}^{*} \cap D^{n}\right\}$ is flat; or (by the second paragraph of this section) that the focal sets $F_{c}$ of $\left\{H_{c}^{*}\right\}$ are all $(k-1)$-planes.

By Theorem 2, a hyperbolic family $\left\{H_{c}\right\}$ generating an immersion of curvature -1 has a unique parallelizing curve in $\bar{H}^{n}$. The following theorem relates the parallelizing curve of $\left\{H_{c}\right\}$ in $\bar{H}^{n}$ to the focal family of $\left\{H_{c}^{*}\right\}$ in $E^{n}$ via the classical notion of duality of points and hyperplanes. For any $p \in \bar{D}^{n}$, let $p^{*}$ denote the inversion of $p$ through $S^{n-1}$; then the hyperplane through $p^{*}$ and orthogonal to the line $O p p^{*}$ is the dual to $p$ through $S^{n-1}$.

THEOREM 7. Let $\left\{H_{c}\right\}_{c \in I}$ be a regular family of hyperbolic $k$-planes which is parallel in $\bar{H}^{n}$ along a curve $\sigma: I \rightarrow \bar{H}^{n}$, and let $\left\{H_{c}^{*}\right\}$ be the associated Euclidean family. Then the focal set $F_{c}$ of $\left\{H_{c}^{*}\right\}$ is the $(k-1)$-plane dual to $\sigma(c)$ through $H_{c}^{*} \cap S^{n-1}$.

Proof. By Theorem 2 and remarks above, the hyperbolic and Euclidean families $\left\{H_{c}\right\}$ and $\left\{H_{c}{ }^{*}\right\}$ have variation fields $l U$ and $l^{*} U^{*}$ respectively at $c$, where $U$ is a covariantly constant unit normal to $H_{c}$, with respect to $g ; U^{*}$ is a constant unit normal to $H_{c}^{*}$, with respect to $g^{*} ; l$ is positive, and hyperbolic on $g$-geodesics; and $l^{*}$ is affine on $g^{*}$-geodesics. Moreover, $U$ and $U^{*}$ are tangent to the same $(k+1)$-plane $P$ through $H_{c}{ }^{*}$. By definition of variation field, the following equation holds on $H_{c}=H_{c}^{*} \cap D^{n}$ :

$$
l^{*}=l U \cdot U^{*} \text {. }
$$

Let $q$ be the center and $r_{1}$ the radius of $H_{c}^{*} \cap D^{n}$, and $r_{2}$ the radius of $P \cap D^{n}$. By (10) and hyperbolicity of $l$, if $p$ is a fixed point of $H_{c}$ then

$$
l^{*}(p)=r_{2}^{-1}\left(a r_{1}^{2}+b r_{1} D^{*}(q, p)\right)
$$

where $a=l(q), b=Y(p) l$ and $Y(p)$ is the $g$-unit vector at $q$ tangent to the $g$-ray $\gamma$ from $q$ toward $p$. Since $l^{*}$ is affine, formula (11) in fact holds at any $p \in H_{c}{ }^{*}$. Therefore we may choose $p$ so that $p \in F_{c}$ and $D^{*}(q, p)$ realizes 
distance from $q$ to $F_{c}$. Then $D^{*}(q, p)=-a b^{-1} r_{1}$ since $l^{*}(p)=0$; and $b(<0)$ is minimized since $D^{*}(q, p)$ is minimized. By definition of $b$, it follows that the ray $\gamma$ from $q$ toward $p$ is tangent to $-\left(\operatorname{grad}_{g} l\right)_{q}$.

Now, $\sigma(c)$ is the striction point of $\left\{H_{c}\right\}$, that is, the minimum point of $i$. Since $l$ is hyperbolic, $\sigma(c)$ clearly lies on this same ray $\gamma$; and since $(l \circ \gamma)(s)$ $=a \cosh s+b \sinh s$, then

$$
D(q, \sigma(c))=\tanh ^{-1}\left(-a^{-1} b\right) \text { and } D^{*}(q, \sigma(c))=-a^{-1} b r_{1} .
$$

Therefore $p$ is the inversion of $\sigma(c)$ through $H_{c}^{*} \cap S^{n-1}$. This completes the proof of Theorem 7.

Note that it is a consequence of Theorem 7 that $\sigma$ is smooth in $\bar{H}^{n}$. The following corollary gives certain properties which $\sigma$ must possess at $H_{\infty}^{n}$. Theorem 8 will show that these are in a sense exhaustive. (In the rest of this section, we use the following convention, based on the canonical identification with $\mathbf{R}^{n}$ of each tangent space to $\mathbf{R}^{n}$. If $\beta$ denotes a Euclidean curve, $\beta^{\prime}(c), \beta^{\prime \prime}(c)$, and $\beta^{(3)}(c)$ denote elements of the tangent space at $\beta(c)$, unless these symbols occur in an algebraic expression, in which case they denote elements of $\mathbf{R}^{n}$.)

COROLlaRY 2. Let $\left\{H_{c}\right\}_{c \in I}$ be a family of hyperbolic k-planes which is parallel in $\bar{H}^{n}$ along a smooth curve $\sigma: I \rightarrow \bar{H}^{n}$, and suppose $\sigma(c) \in H_{\infty}^{n}$ for some $c$. Then $\sigma^{\prime}(c)$ is tangent to $H_{c \infty}$, and $\sigma^{\prime \prime}(c)$ is tangent to $\bar{H}_{c}$.

Proof. Note that we allow the possibility that $\left\{H_{c}\right\}$ is not regular at $c$, in which case the variation fields of both $\left\{H_{c}\right\}$ and $\left\{H_{c}^{*}\right\}$ vanish. By Theorem 7 (or trivially, in the nonregular case), $\sigma(c)$ lies in the focal set $F_{c}$ of $\left\{H_{c}^{*}\right\}$, and so the variation field for $\left\{H_{c}^{*}\right\}$ vanishes at $\sigma(c)$. It follows from the definition of variation field and the fact that $\sigma(d) \in H_{d}^{*}$ for all $d \in I$ that $\sigma^{\prime}(c)$ is tangent to $H_{c}^{*}$. Since $\sigma$ is not transverse to $S^{n-1}, \sigma^{\prime}(c)$ is tangent to $H_{c \infty}=H_{c}^{*} \cap S^{n-1}$.

Denote by $\sigma^{*}(d)$ the inversion of $\sigma(d)$ through $H_{d}^{*} \cap S^{n-1}$. By Theorem 7, $\sigma^{*}(d) \in F_{d}$ for all $d \in I$, hence $\sigma^{* \prime}(d)$ is tangent to $H_{d}^{*}$. In particular, since $\sigma^{*}(c)=\sigma(c)$ and $\sigma^{*}$ is not transverse to $S^{n-1}, \sigma^{* \prime}(c)$ is tangent to $H_{c}^{*} \cap S^{n-1}$, and thus to $F_{c}$ (since either $F_{c}=H_{c}^{*}$ or $F_{c}$ is the hyperplane of $H_{c}^{*}$ tangent to $S^{n-1}$ at $\sigma(c)$ ). Setting $\zeta=\sigma^{*}+\sigma^{* \prime}$, we have $\zeta(d) \in H_{d}^{*}$ for all $d$, and $\zeta(c) \in F_{c}$. Therefore $\zeta^{\prime}(c)$ is tangent to $H_{c}^{*}$. But $\sigma^{* \prime \prime}(c)=\zeta^{\prime}(c)-$ $\sigma^{* \prime}(c)$, so $\sigma^{* \prime \prime}(c)$ is also tangent to $H_{c}^{*}$. Moreover, it may be verified by direct computation that because $\sigma$ and $\sigma^{*}$ are inverse curves and $\sigma^{* \prime \prime}(c)$ is tangent to $H_{c}^{*}$, then $\sigma^{\prime \prime}(c)$ is tangent to $H_{c}^{*}$. Corollary 2 follows.

We are interested in regular families, since they always generate submanifolds. The following condition will be useful:

Corollary 3. A parallel family $\left\{H_{c}\right\}$ along a regular curve $\sigma: I \rightarrow \bar{H}^{n}$ is 
regular if and only if $\sigma^{\prime}(c)$ is transverse to $H_{c}$ whenever $\sigma(c) \in H_{c}$ and $\sigma^{(3)}(c)$ is transverse to $\bar{H}_{c}$ whenever $\sigma(c) \in H_{c \infty}$.

Proof. (Note that by Theorem 2, for a parallel family regularity is equivalent to having no focal points.) When $\sigma$ lies in $H^{n}$, the proof is the same as for Theorem 4.1. If $\sigma(c) \in H_{\infty}^{n}=S^{n-1}$, then $\sigma \cdot \sigma^{\prime \prime}<0$ at $c$ because $\sigma$ is regular; therefore $\sigma(c)+k \sigma^{\prime \prime}(c)$ lies in $D^{n}$ for some $k>0$, and hence in $H_{c}=H_{c}^{*} \cap D^{n}$ by Corollary 3.2. Now consider the curve $\zeta=\sigma+k \sigma^{\prime \prime}$. If $\sigma^{(3)}(c)$ is transverse to $H_{c}^{*}$, then so is $\zeta^{\prime}(c)$, and it follows that $\left\{H_{c}\right\}$ is regular at $c$. If $\sigma^{(3)}(c)$ is tangent to $H_{c}^{*}$, then $\left\{H_{c}\right\}$ focuses at $\zeta(c)$, and so $\left\{H_{c}\right\}$ is not regular at $c$. Thus Corollary 3 is proved.

A parallelizing curve $\sigma$ in $\bar{H}^{n}$ of a regular family of $k$-planes, while always regular where $\sigma$ lies in $H^{n}$, need not be regular at $H_{\infty}^{n}$. The regularity hypothesis on $\sigma$ is necessary for the two uniqueness results which follow. Note that for a regular curve in $D^{n}$, the osculating 2-planes with respect to $g$ and $g^{*}$ agree, when they exist. Furthermore, for a regular curve in $\bar{D}^{n}$ and lying on $S^{n-1}$ at $c$, the osculating 2-plane at $c$ with respect to $g^{*}$ exists and enters $D^{n}$. Therefore one may speak without ambiguity of the osculating hyperbolic 2-plane at $c$ of any regular curve $\sigma$ in $\bar{H}^{n}$ such that $\sigma(c) \in H_{\infty}^{n}$. For any parallel family along $\sigma$, the $k$-plane at $c$ must contain this 2-plane, by Corollary 2.

THEOREM 8. Let $\sigma: I \rightarrow H_{\infty}^{n}$ be a regular curve, where $0 \in I$, and $H_{0}$ be any hyperbolic $k$-plane containing the osculating hyperbolic 2-plane of $\sigma$ at $\sigma(0)$. Then $H_{0}$ extends uniquely to a parallel family $\left\{H_{c}\right\}_{c \in I}$ along $\sigma$.

Proof. We reduce to a linear problem, that of parallel translation in the Euclidean normal bundle of $\sigma$ (regarded as a curve lying on $S^{n-1}$ ). If $\left\{H_{c}\right\}$ is parallel along $\sigma$, then the corresponding Euclidean family $\left\{H_{c}^{*}\right\}$ has the following properties (by Corollary 2 and Theorem 7):

(i) $H_{c}^{*}$ contains the osculating 2-plane of $\sigma$ at $\sigma(c)$, and

(ii) the focal set $F_{c}$ is either $H_{c}{ }^{*}$ or the hyperplane of $H_{c}{ }^{*}$ tangent to $S^{n-1}$ at $\sigma(c)$.

Conversely, if $\left\{H_{c}^{*}\right\}$ is a Euclidean family satisfying (i) and (ii), then each $H_{c}^{*}$ enters $D^{n}$ by (i), and we claim that the corresponding hyperbolic family $\left\{H_{c}\right\}$ is parallel along $\sigma$. Where the families are not regular, constancy of the hyperbolic variation field on horospheres is obvious. Elsewhere, since each $F_{c}$ is a $(k-1)$-plane not entering $D^{n},\left\{H_{c}\right\}$ generates a submanifold of curvature -1 in $H^{n}$; thus by Theorems 2 and $7,\left\{H_{c}\right\}$ is parallel along $\sigma$. Therefore it suffices, for the theorem, to demonstrate existence and uniqueness of a family $\left\{H_{c}^{*}\right\}$ of Euclidean $k$-planes satisfying (i) and (ii), when $H_{0}^{*}$ is given.

Suppose such a family $\left\{H_{c}^{*}\right\}$ exists. Let $\mathcal{T}$ be the Euclidean vector bundle over $\sigma$ whose $(k-2)$-dimensional fiber at each $c$ is tangent to $H_{c}{ }^{*} \cap S^{n-1}$ 
and orthogonal to $\sigma^{\prime}(c)$. Then $H_{c}{ }^{*}$ is tangent to $\left[{ }^{\alpha}, \sigma^{\prime}, \sigma^{\prime \prime}\right]_{c}$ (where bracket denotes span). Let $v$ be a smooth section of $\mathcal{T}$. Setting $\zeta=\sigma+v$, we have $\zeta(c) \in F_{c}$ for all $c$, hence $\zeta^{\prime}(c)$ tangent to $H_{c}{ }^{*}$. Therefore $v^{\prime}(c) \in\left[\mathcal{T}^{\top}, \sigma^{\prime}, \sigma^{\prime \prime}\right]_{c}$. But $v^{\prime} \cdot \sigma=0$ since $v \cdot \sigma=v \cdot \sigma^{\prime}=0$, and so $v^{\prime}(c) \in\left[\mathcal{T}^{\prime}, \sigma^{\prime}\right]_{c^{\prime}}$. Therefore $\widetilde{T}^{*}$ has parallel fiber in the Euclidean normal bundle of $\sigma$. Since $\tau^{\circ}$ is uniquely determined by $H_{0}{ }^{*}$ and $\sigma$, so is $\left\{H_{c}^{*}\right\}$.

On the other hand, for given $H_{0}{ }^{*}$ let $\Upsilon_{0}$ consist of the vectors at $\sigma(0)$ tangent to $H_{0}{ }^{*} \cap S^{n-1}$ and orthogonal to $\sigma$, and $\Upsilon$ be the parallel extension of $\Upsilon_{0}$ in the Euclidean normal bundle of $\sigma$. Then $\Upsilon^{\top}$ is always tangent to $S^{n-1}$. Letting $H_{c}{ }^{*}$ be the $k$-plane through $\sigma(c)$ tangent to $\left[{ }^{\circ}, \sigma^{\prime}, \sigma^{\prime \prime}\right]_{c}$, we have a family satisfying (i). For (ii), it suffices to verify that $\left[\mathcal{T}^{\top}, \sigma^{\prime}\right]_{c}$ is always tangent to the focal set $F_{c}$ of $\left\{H_{c}^{*}\right\}$. But for any curve of the form $\zeta=\sigma+v+f \sigma^{\prime}$ with $v$ a section of $\mathfrak{V}$, then $\zeta^{\prime}(c) \in\left[\mathcal{V}, \sigma^{\prime}, \sigma^{\prime \prime}\right]_{c}$ and so $\zeta^{\prime}(c)$ is tangent to $H_{c}^{*}$. Therefore $\zeta(c) \in F_{c}$, as required. Thus we have constructed a family $\left\{H_{c}^{*}\right\}$ satisfying (i) and (ii).

Corollary 4. Let $\sigma: I \rightarrow \bar{H}^{n}$ be a regular curve, where $0 \in I$, and $H_{0}$ be a hyperbolic $k$-plane at $\sigma(0)$. If there exists a parallel family along $\sigma$ extending $H_{0}$, then that family is unique.

Proof. Note that if two hyperbolic $k$-planes intersect, one may speak of the hyperbolic angle between them; this angle is measured in the $(n-r)$-plane which is $g$-orthogonal to the intersection $r$-plane $P$ at any $p \in P$, and is independent of the choice of $p$. We shall show that any two parallel families $\left\{H_{1 c}\right\}$ and $\left\{H_{2 c}\right\}$ of $k$-planes along a given regular curve $\sigma: I \rightarrow \bar{H}^{n}$ have constant intersection dimension, and form a constant angle.

Note that $H_{1 c} \cap H_{2 c} \neq \varnothing$. Indeed, if $\sigma(c) \in H^{n}$ then $\sigma(c)$ lies in this intersection, and if $\sigma(c) \in H_{\infty}^{n}$ then this intersection contains a 2-plane at least. Let $I_{0}$ be a maximal subinterval of $I$ on which the intersection dimension takes its minimum for $I$. Then $I_{0}$ is open in $I$. The angle between the two families is smooth on $I_{0}$, and certainly is constant on any subinterval on which $\sigma$ lies entirely in $H^{n}$. Furthermore, it follows from the proof of Theorem 8 that the angle is constant on any subinterval $J$ of $I_{0}$ on which $\sigma$ lies entirely in $H_{\infty}^{n}$ : In the notation of that proof, suppose $H_{i c}$ corresponds to $\left[\mathscr{V}_{i}, \sigma^{\prime}, \sigma^{\prime \prime}\right]_{c}$, for each $c \in J$. It may be verified that the hyperbolic angle between $H_{1 c}$ and $H_{2 c}$ is equal to the Euclidean angle between $\left[\mathcal{T}_{1}, \sigma^{\prime}\right]_{c}$ and $\left[\mathscr{V}_{2}, \sigma^{\prime}\right]_{c}$, since $\left[\mathscr{V}_{i}, \sigma^{\prime}\right]_{c}$ is tangent to $H_{i c}^{*} \cap S^{n-1}$ at $\sigma(c)$. In turn, the Euclidean angle between the $\left[\mathcal{V}_{i}, \sigma^{\prime}\right]$ equals that between the $\mathcal{\Upsilon}_{i}$ since the $\mathcal{T}_{i}$ are orthogonal to $\sigma^{\prime}$. This angle is constant on $J$ because the $\widetilde{T}_{i}$ are parallel in the Euclidean normal bundle of $\sigma \mid J$.

Therefore the hyperbolic angle between the two families has vanishing 
derivative on a dense subset of $I_{0}$, hence is constant on $I_{0}$. It follows that $I_{0}$ is closed in $I$, and $I_{0}=I$.

REMARK 6. Given a regular curve $\sigma: I \rightarrow \bar{H}^{n}$, and an initial $k$-plane $H_{0}$, there may be no parallel family on $\sigma$ extending $H_{0}$. For example, if $\sigma(0) \in$ $H^{n}$, the the parallel translate of $H_{0}$ along $\sigma$ as $\sigma$ approaches $H_{\infty}^{n}$ may have more than one limit $k$-plane, or none at all.

5. Examples. By way of illustration, we examine families of hyperbolic $k$-planes which have parallelizing curves in $\bar{H}^{n}$ of special type. Our conventions are the same as in $\$ 4$.

EXAMPLE 2. A family $\left\{H_{c}\right\}_{c \in I}$ without focal points is parallel along an orthogonal trajectory $\sigma: I \rightarrow H^{n}$ if and only if the corresponding Euclidean $k$-planes $H_{c}^{*}$ all pass through a fixed $(k-1)$-plane not meeting $\bar{D}^{n}$. This may be seen as follows.

Note that if $\sigma$ is both parallelizing and orthogonal, then $\sigma$ lies in a hyperbolic $(n-k)$-plane orthogonal to the $H_{c}$. To see this, observe that the family $\left\{H_{c}{ }^{\perp}\right\}$ of hyperbolic $(n-k)$-planes, where $H_{c}{ }^{\perp} \cap H_{c}=\sigma(c)$ and $H_{c}{ }^{\perp}$ is orthogonal to $H_{c}$, is parallel along and tangent to $\sigma$. Thus the variation field at $c$ of $\left\{H_{c}{ }^{\perp}\right\}$ vanishes at $\sigma(c)$, and, by Theorem 2, vanishes identically. Therefore the $H_{c}{ }^{\perp}$ coincide, in an $(n-k)$-plane containing $\sigma$. Now, it can be shown that a hyperbolic $k$-plane is orthogonal to a given hyperbolic $(n-k)$ plane $H$ if and only if the corresponding Euclidean $k$-plane lies in the pencil through a certain Euclidean $(k-1)$-plane, not meeting $\bar{D}^{n}$, determined by $H$. Thus, since the $H_{c}$ are all orthogonal to a fixed $(n-k)$-plane, it follows that the $H_{c}{ }^{*}$ all pass through a fixed $(k-1)$-plane.

The converse follows from Theorem 7 and the fact that, if $\left\{H^{*}\right\}$ now denotes the collection of Euclidean $k$-planes passing through a fixed $(k-1)$-plane $F$ and entering $D^{n}$, the dual points of $F$ in all the $H^{*}$ form a hyperbolic $(n-k)$-plane which is $g$-orthogonal to each $H=H^{*} \cap D^{n}$.

EXAMPLE 3. A family $\left\{H_{c}\right\}$ has constant parallelizing curve in $H_{\infty}^{n}$ if and only if the $H_{c}^{*}$ all pass through a fixed $(k-1)$-plane tangent to $S^{n-1}$. Here it suffices to verify that if the parallelizing curve $\sigma$ is constant at $H_{\infty}^{n}$, then the focal family $\left\{F_{c}\right\}$ of $\left\{{H_{c}}^{*}\right\}$ is constant. If $\left\{F_{c}\right\}$ is not constant, then at some $c$ the variation field of $\left\{F_{c}\right\}$ does not vanish identically on $F_{c}$. This field is defined on the hyperplane $F_{c}$ of $H_{c}{ }^{*}$ and is tangent to $H_{c}{ }^{*}$. Since all $F_{c+\delta}$ lie in the tangent plane to $S^{n-1}$ at the image point of $\sigma$, it follows that $H_{c}^{*}$ is tangent to $S^{n-1}$, which is impossible since $H_{c}^{*}$ enters $D^{n}$.

EXAMPLE 4. The osculating Euclidean 2-planes of a regular curve $\sigma$ lying on $S^{n-1}$ and having nowhere vanishing torsion (second order curvature function) correspond to a regular family of hyperbolic 2-planes which is parallel along $\sigma$. Indeed, parallelism along $\sigma$ follows from the proof of Theorem 8. Regularity is a consequence of Corollary 3 and the torsion 
assumption. Conversely, all regular hyperbolic 2-plane families which are parallel along a regular curve in $H_{\infty}^{n}$ are obtained in this way, by Corollaries 2 and 3.

Now suppose $n=3$, and consider all foliations of $H^{3}$ by 2-plane families whose parallelizing curves are regular curves in $H_{\infty}^{3}$. These foliating families correspond precisely to the families of osculating 2-planes of regular Euclidean curves lying on $S^{2}$, for which torsion is nonvanishing and curvature (denoted here by $\kappa^{*}$ ) becomes infinite at both endpoints. For example, a loxodrome is such a curve. It is only necessary to verify that for a unit-speed curve $\sigma$ on $S^{2}$, the osculating planes approach tangency to $S^{2}$ at an endpoint if and only if curvature becomes infinite. This follows by differentiating $\sigma \cdot \sigma^{\prime}=0$, to obtain $\left|\sigma \cdot \sigma^{\prime \prime} /\left\|\sigma^{\prime \prime}\right\|\right|=1 / \kappa^{*}$.

EXAMPLE 5. Let $\left\{H_{c}^{*}\right\}$ be any regular family of $(n-1)$-planes in $E^{n}$ such that each member intersects $D^{n}$. The equation of $H_{c}^{*}$ is $y \cdot N(c)-r(c)=0$, where $N$ and $r$ denote (smoothly varying) unit normal and distance from the origin respectively. Then the focal $(n-2)$-plane $F_{c}$ in $H_{c}^{*}$ satisfies $y \cdot N^{\prime}(c)$ $-r^{\prime}(c)=0$. (Indeed, $y \in F_{c}$ if and only if there is a curve $\beta$ satisfying $\beta(c)=y, \beta(c+\delta) \in H_{c+\delta}^{*}$, and $\beta^{\prime}(c) \cdot N(c)=0$; but the last two conditions imply $\beta(c) \cdot N^{\prime}(c)-r^{\prime}(c)=0$.) If we assume that $\left\{F_{c}\right\}$ never enters $D^{n}$, then $\left\{H_{c}^{*}\right\}$ determines a regular hyperbolic family which is parallel along some curve $\sigma$ in $\bar{H}^{n}$. By Theorem 7 , the equations for $\left\{F_{c}\right\}$ yield a formula for $\sigma$ :

$$
\sigma=r N+\left(1-r^{2}\right)\left(r^{\prime}\right)^{-1} N^{\prime} .
$$

Here $r^{\prime}$ never vanishes because $\left\{F_{c}\right\}$ never enters $D^{n}$.

This formula may be used to construct examples, such as the following one for $n=2$. The curve $\sigma^{*}(c)=\left(1+c^{2}, c^{3}\right)$ lies outside $S^{1}$ and meets $S^{1}$ at $c=0$, in a cusp tangent to the $x$-axis. The tangent lines $H_{c}^{*}$ to $\sigma^{*}$ form a regular Euclidean family (even though $\sigma^{*}$ is not regular). The focal sets of $\left\{H_{c}^{*}\right\}$ are given by $F_{c}=\sigma^{*}(c)$, and hence lie outside $D^{2}$. Thus for a certain symmetric interval $I$ about 0 , the tangent lines to $\sigma^{*} \mid I$ determine a family $\left\{H_{c}\right\}$ of hyperbolic lines foliating $H^{2}$. The parallelizing curve $\sigma$ may be computed from (12) to be

$$
\sigma(c)=\left(3 c^{4}+2 c^{2}+2\right)^{-1}\left(c^{6}+3 c^{4}+2,-c^{5}-4 c^{3}\right) .
$$

Note that $\sigma^{\prime}(0)=0$, as required by Corollary 3, and that $\sigma$ meets $S^{1}$ at $c=0$, in a cusp tangent to the $x$-axis.

REMARK 7. Finally, we mention one further possible direction for investigation: With regard to $\$ 4$, one might ask precisely which curves $\sigma: I \rightarrow \bar{H}^{n}$ (taking values in both $H^{n}$ and $H_{\infty}^{n}$ ) allow the existence of parallel families. 


\section{REFERENCES}

1. D. Ferus, On isometric immersions between hyperbolic spaces, Math. Ann. 205 (1973), 193-200.

2. P. Hartman and L. Nirenberg, On spherical image maps whose Jacobians do not change sign, Amer. J. Math. 81 (1959), 901-920.

3. S. Kobayashi and K. Nomizu, Foundations of differential geometry, vol. 1, Interscience, New York, 1963.

4. K. Nomizu, Isometric immersions of the hyperbolic plane into the hyperbolic space, Math. Ann. 205 (1973), 181-192.

5. B. O'Neill and E. Stiel, Isometric immersions of constant curvature manifolds, Michigan Math. J. 10 (1963), 335-339.

6. E. Portnoy, Developable surfaces in hyperbolic space, Pacific J. Math. 57 (1975), 281-288.

Department of Mathematics, University of Illinois, Urbana, Illinois 61801 